\title{
Continuous Cropping Regulates Fungal Community Structures and Functional Groups in Rhizosphere Soil of Tibet Barley
}

\section{Youhua Yao}

Qinghai University

Yuan Zhao

Qinghai University

Xiaohua Yao

Qinghai University

Yixiong Bai

Qinghai University

Likun An

Qinghai University

Xin Li

Qinghai University

Kunlun Wu ( $\nabla$ wklqaaf@sina.com )

Qinghai University

\section{Research Article}

Keywords: Tibet barley, continuous cropping, rhizosphere soil, fungal community, ecologically functional group

Posted Date: November 20th, 2020

DOI: https://doi.org/10.21203/rs.3.rs-109459/v1

License: (c) (1) This work is licensed under a Creative Commons Attribution 4.0 International License. Read Full License 


\section{Abstract}

Continuous cropping regulates the community structure of rhizosphere soil microbes; however, the effects of continuous cropping on the community structure and function of rhizosphere fungal communities of plants cultivated in high-altitude regions are not well understood. In this study, 18S rRNA gene highthroughput sequencing was applied to examine the rhizosphere fungal community structure during continuous cropping of Tibet barley (a principal cereal cultivated on the Qinghai-Tibetan Plateau). The results showed that the Chao1 and phylogenetic diversity (PD) indices declined as cropping years increased. Additionally, relative abundance of the genera Cystofilobasidium, Mucor, and Ustilago increased, whereas the abundance of Fusarium decreased during continuous cropping. Furthermore, identification of ecological groups using FUNGuild revealed that saprotrophs, pathogens, and symbiotrophs were the dominant groups in rhizosphere soil, and these three trophic modes all increased significantly with continuous cropping. During continuous cropping, the fungal plant-pathogens Parastagonospora and Ustilago also increased remarkably, as did the endophyte fungi Verticillium. Collectively, continuous cropping of Tibet barely increased the potential for plant-pathogenic and endophyte fungi in rhizosphere soil. Thus, the development of sustainable farming practices to reduce the abundance of harmful fungi is vital to Tibet barley growth and production during continuous cropping of plants cultivated in high-altitude regions.

\section{Introduction}

Continuous cropping, which is the agricultural practice of long-term cultivation of the same crop in identical soil, is a vital agricultural issue because it leads to reduced the soil quality and augmentation of harmful pests and soilborne microbial communities, resulting in yield decline of plants ${ }^{1}$. Rhizosphere soil microorganisms affect the growth, development, and health status of plants ${ }^{2}$, and the influences of continuous cropping on microbial community structures in rhizosphere soil have gained considerable attention $^{3-7}$. When compared with bacteria, rhizosphere fungi are important decomposers that play vital roles in balancing the rhizosphere ecosystem ${ }^{8}$. Moreover, many fungi are closely associated with plant diseases, and research has shown that accumulation of fungal pathogens is often responsible for continuous cropping problems ${ }^{9}$.

High-altitude ecosystems are generally characterized by low temperature, variable rainfall, reduced atmospheric pressure, and soil nutritional stress ${ }^{10}$. In high-altitude regions, low temperature is the major factor influencing microbial diversity and function ${ }^{11}$. Plants and microbes have co-evolved and interact with each other in the environment; thus, high-altitude regions are important to understanding the interactions between certain microbes and plants cultivated in cold environments ${ }^{12}$. Moreover, lower species richness and diversity have been observed in soil from higher elevations ${ }^{13,14}$. In a study of three vertical climate zones on the Qinghai-Tibetan Plateau, China (av. 4,000 m above sea level), soil bacteria exhibited more apparent elevational zonation features and decreased diversity patterns with increasing elevation ${ }^{14}$. Studies have also been conducted to explore the dynamics of rhizosphere fungal 
communities under continuous cropping of plants cultivated at normal altitudes, such as soybean ${ }^{15}$, potato ${ }^{5}$, strawberry ${ }^{16}$, peanut ${ }^{17}$, tea ${ }^{18}$, and cucumber ${ }^{19}$. However, the fungal community structures of rhizosphere soil in high altitude regions are less understood. Additionally, changes in potentially beneficial and pathogenic fungi have not been studied during continuous cropping of plants cultivated in high-altitude regions.

Tibet barley (Hordeum vulgare L., qingke), which serves as a staple food and an important livestock feed in the Tibetan Plateau ${ }^{20}$, is drought and cold tolerant, exhibiting strong adaptability to the extreme climate of the plateau ${ }^{21}$. The cropping area of Tibet barely accounts for approximately $43 \%$ of the grain crop area on the Tibetan Plateau ${ }^{22}$. We previously found that continuous cropping reduced the yield of Tibet barley (accepted data). Thus, understanding the influence of continuous cropping on the fungal community in the soil rhizosphere is essential to designing effective farm management practices to relieve soil depletion associated with Tibetan barley cultivation. Therefore, we assume that the continuous cropping of Tibet barley may directly influence the diversity and function of fungal communities. In this study, we considered the fungal communities in Tibet-barley fields with 2 to 6 years of monoculture history. The objectives of our study were to explore the dynamics of diversity and composition of the rhizosphere fungal community composition during continuous cropping. Furthermore, we evaluated changes in fungal groups with potential ecological functions during continuous cropping. The results presented herein will provide a better understanding of the response of rhizosphere soil fungal communities to continuous cropping of plants cultivated in high-altitude regions.

\section{Results}

\section{Dynamics of fungal community diversity and composition}

A total of 783,935 high-quality sequences of $18 \mathrm{~S}$ rRNA were obtained and each sample was evenly rarefied to 38,031 reads before diversity calculation. Continuous cropping was found to have a negative effect on the species richness and phylogenetic diversity of rhizosphere fungal communities of Tibet barley. The Chao1 and PD indices of the rhizosphere fungal community significantly decreased from 2 to 5 years, but slightly increased after 5 years of continuous cropping (Table 1). The Chao 1 index decreased from $301.5 \pm 10.23$ at 2 years to $224.8 \pm 6.6$ at 5 years of continuous cropping, then climbed slightly to $233.0 \pm 21.3$ after 6 years. The PD indices decreased from $19.8 \pm 0.6$ to $13.4 \pm 0.5$, then increased slightly to $14.5 \pm 1.1$ after 6 years. However, no distinct pattern of species diversity (represented by Shannon and Simpson indices) was observed during continuous cropping (Table 1). PCoA was used to depict the degree of fungal community differentiation after continuous cropping for various lengths of time. The first two principal coordinates explained $60.31 \%$ of the total variance of the rhizosphere soil fungal community structures (Figure 1). PCoA revealed that fungal communities from the second and third years (CC2Y and CC3Y) were separated from those from later years (CC4Y, CC5Y, and CC6Y) along the first PCoA axis. Communities from the last three years were separated along the second PCoA axis (Figure 1). 
Table 1. Effects of continuous cropping on fungal alpha diversities after different continuous cropping durations*.

\begin{tabular}{cccccc}
\hline Diversity & \multicolumn{5}{c}{ Duration of continuous cropping (years) } \\
\cline { 2 - 6 } Indice & 2 & 3 & 4 & 5 & 6 \\
\hline Chao1 & $301.75 \pm 9.84^{\mathrm{a}}$ & $259.25 \pm 7.41^{\mathrm{b}}$ & $239.25 \pm 13.48^{\mathrm{bc}}$ & $224.25 \pm 6.24^{\mathrm{c}}$ & $231.50 \pm 20.87^{\mathrm{bc}}$ \\
PD & $19.64 \pm 0.74^{\mathrm{a}}$ & $16.12 \pm 0.28^{\mathrm{b}}$ & $14.51 \pm 0.10^{\mathrm{c}}$ & $13.16 \pm 0.50^{\mathrm{c}}$ & $14.22 \pm 1.06^{\mathrm{c}}$ \\
Shannon & $5.99 \pm 0.04^{\mathrm{b}}$ & $5.72 \pm 0.30^{\mathrm{ab}}$ & $6.19 \pm 0.15^{\mathrm{ab}}$ & $6.28 \pm 0.09^{\mathrm{a}}$ & $6.18 \pm 0.14^{\mathrm{ab}}$ \\
Simpson & $0.95 \pm 0.00^{\mathrm{b}}$ & $0.94 \pm 0.02^{\mathrm{ab}}$ & $0.97 \pm 0.00^{\mathrm{a}}$ & $0.98 \pm 0.00^{\mathrm{a}}$ & $0.97 \pm 0.00^{\mathrm{a}}$ \\
\hline
\end{tabular}

* Each slample was evenly rarefied to 38,031 reads before diversity calculation. Values represent means \pm standard deviation $(n=4)$. Different lowercase letters within the same column indicate significant differences among different continuous cropping durations at $P<$ 0.05 according to two-way analysis of variance.

Nearly $79 \%$ of the total sequences of the 18 S rRNA gene could be taxonomically classified at the phylum level. Ascomycota (56.74\% $\pm 4.91 \%)$, Basidiomycota (17.45\% $\pm 7.60 \%)$, and Mucoromycota $(3.21 \% \pm 1.91 \%)$ were the dominant phyla (Figure S1), accounting for more than $75 \%$ of all sequences. The predominant families in the rhizosphere soil were Nectriaceae, Mucoraceae, and Pleosporaceae (Figure 2A).

Nectriaceae was the only abundant family that showed a decreasing trend (from $10.06 \% \pm 0.71 \%$ to $3.28 \% \pm 0.14 \%$ ) throughout the experiment. The abundances of all other dominant families (Cystofilobasidiaceae, Mucoraceae, Phaeosphaeriaceae, Pleosporaceae, Sarcosomataceae, Sarocladiaceae, and Ustilaginaceae) increased during continuous cropping. Among these, Ustilaginaceae increased 46.8 -fold, from $0.12 \% \pm 0.02 \%$ to $5.62 \% \pm 1.02 \%$ (Figure $2 \mathrm{~A}$ ). At the genus level, Fusarium, Mucor, Parastagonospora, Sarocladium, and Ustilago mostly appeared across all samples (Figure 2B). The relative abundance of Fusarium fluctuated, but showed an overall decrease from $10.06 \% \pm 0.71 \%$ to $3.28 \% \pm 0.14 \%$ throughout the experiment. Cystofilobasidium and Ustilago were most abundant after 6 years of continuous cropping (Figure 2B).

\section{Taxa shared in rhizosphere soil during continuous cropping}


Shared taxa were classified at the phylum, class, order, and genus level for all samples. Taxa that showed more or less than $1 \%$ mean relative abundance were defined as dominant or rare, respectively (Figure 3 ). Among the rhizosphere fungal communities, 42 shared genera were identified during continuous cropping, comprising about $31.82 \%$ (dominant shared genus, $\sim 16.67 \%$, rarely shared genus, $\sim 15.15 \%$ ) of the total sequence abundance (Figure 3). Fusarium was the only dominant shared genera identified in all samples with different years of continuous cropping, while Parastagonospora, Sarocladium, Tetracladium, and Cystofilobasidium were the dominant shared genera after 4 years of cropping (Figure 4).

\section{Functional group succession during continuous cropping}

The functions of the soil fungal community were classified into ecological guilds using FUNGuild ${ }^{23}$. There were 132 OTUs, which accounted for $19.7 \%$ of the total sequences that could be allocated into fungal functional groups using the FUNGuild annotation tool. Among assigned OTUs with function, saprotrophs were the dominant trophic type, followed by pathogens, and then symbiotrophs (Figure S2). The relative abundances of saprotrophs, pathogens, and symbiotrophs all increased significantly with continuous cropping. Specifically, saprotrophs increased from $7.73 \% \pm 0.24 \%$ to $14.90 \% \pm 1.10 \%$, pathogens from $4.88 \% \pm 0.33 \%$ to $15.47 \% \pm 0.79 \%$, and symbiotrophs from $1.83 \% \pm 0.01 \%$ to $5.97 \% \pm$ $0.44 \%$ (Figure S2). In the pathogen guild, plant pathogens were most abundant and continuously increased from $2.76 \% \pm 0.18 \%$ to $13.20 \% \pm 1.94 \%$. Furthermore, animal pathogens, fungal parasites, and lichen parasites all increased after continuous cropping (Figure 5A). Endophytes and undefined saprotrophs were the predominate trophic modes among symbiotrophs and saprotrophs, respectively. The detected guilds of endophytes and undefined saprotrophs also showed increasing trends during continuous cropping (Figure 5B and Figure 5C).

We next focused on the taxonomy of plant pathogens and endophytes because of their high abundance and remarkable increases during continuous cropping (Figure 6A and Figure 6B). Among plant pathogens, unclassified, Parastagonospora, Ustilago, and Verticillium were the dominant genera, and these increased significantly, especially Ustilago, which showed an increase in abundance of approximately 44 -fold, from $1.26 \%$ $\pm 0.24 \%$ o to $55.29 \%$ o $\pm 10.27 \%$ o (Figure $6 \mathrm{~A}$ ). Moreover, the occurrence of Parastagonospora and Verticillium increased by approximately two-fold and three-fold, respectively (Figure 6A). Among endophytes, Verticillium was the only genera that showed an average abundance higher than $1 \%$ of the total sequences, increasing from $2.91 \%$ o $\pm 0.20 \%$ o to $8.28 \%$ o \pm 0.96\%o (Figure 6B).

\section{Discussion}

\section{Continuous cropping influenced fungal diversity and taxonomy in thizosphere soil}

Continuous cropping in the same soil may lead to cumulative effects that could affect fungal diversity. In the present study, we found that continuous cropping of Tibet barley resulted in a remarkable change in 
soil fungal community diversity and richness. The Chao1 and PD indices decreased as the number of crop years increased (Table 1). Similar results have also been observed in rhizosphere soils of tea ${ }^{24}$, strawberry ${ }^{16}$, American ginseng ${ }^{25}$, and sweet potato ${ }^{5}$. Decreases in fungal diversity and richness have been recognized as an essential threat to the ecosystem, resulting in loss of soil function, which may have a negative effect on plant production during continuous cropping ${ }^{26,27}$.

Ascomycota and Basidiomycota, which have been identified as the most abundant phyla in rhizosphere soils of Tibet barley (Figure S1), are reportedly the main decomposers in agricultural waste

composting ${ }^{28,29}$. At the genus level, the abundance of Fusarium decreased with increased cropping years, while the abundance of Ustilago increased tremendously after 6 years of continuous cropping (Figure 2B). In soil, the genus Fusarium comprises many species of environmental, agricultural, and human health importance, but its notoriety mainly results from its pathogenicity toward a wide range of plants ${ }^{30}$. Continuous cropping of soybean increased the relative abundance of Fusarium oxysporum in soil ${ }^{6}$, and Fusarium was linked to soil sickness during cucumber continuous cropping ${ }^{19}$. Moreover, Coptis chinensis monoculture was found to increase Fusarium, which, in turn, promoted root rot disease and limited crop yield ${ }^{7}$. Ustilago, which are potential plant pathogens found in soil, were found to increase after continuous soybean cropping ${ }^{15}$. The divergence between Fusarium and Ustilago may reflect their adaption to changed rhizosphere soil during continuous cropping of Tibet barley.

\section{Effects of continuous cropping on functional groups of the rhizosphere fungal community}

We speculated that continuous cropping of Tibet barley might select specific functional groups as they adapt to root exudates and residues in the rhizosphere. Numerous studies have reported that continuous cropping led to the accumulation of organic acids and phenolic acids secreted by roots, promoting the growth of pathogenic microorganisms and further influencing the structure of the rhizospheric soil microbial community ${ }^{31}$. Our results showed that the continuous cropping of Tibet Barley significantly influenced fungal functions in rhizosphere soil. FUNGuild fungal functional prediction revealed that saprotrophs were the dominant trophic type in rhizospheric fungal communities of the Tibet barley plants in continuous cropping fields (Figure S2), which was similar to the results of a study of Coptis chinensis ${ }^{3}$. These findings could be attributed to the central roles of saprotrophs in organic decomposition during continuous cropping ${ }^{23}$. Generally, symbiotrophic fungi are extremely beneficial to the health, nutrition, and quality of most crops ${ }^{32,33}$. However, pathotrophic fungi usually obtain nutrients by attacking host cells; thus, they are considered to cause disease or have negative effects on plant performance ${ }^{34}$. In this study, the abundances of pathogens increased significantly with continuous cropping (Figure S2). Among pathotrophs, plant pathogens increased with increased continuous cropping time (Figure 5A). These results were consistent with the findings reported by ${ }^{17}$, who stated that continuous cropping increased the abundance of pathogenic fungi in peanut soil. Similarly, the abundances of soil-borne pathogens were increased in continuous cropping fields of cucumber ${ }^{35}$, tomato ${ }^{36}$, and potato ${ }^{37}$. These findings suggested that the environmental conditions under continuous cropping were likely to facilitate pathogen proliferation, and that Tibet barley plants might suffer a higher risk of fungal diseases with extended 
continuous cropping years. Specifically, we found that the relative abundances of the plant pathogens Parastagonospora and Ustilago were significantly increased during continuous cropping of Tibet barley (Figure 6A). Parastagonospora nodorum causes the wheat disease Septoria nodorum blotch and is responsible for substantial yield losses in many regions around the world ${ }^{38}$. As previously reported, corn smut diseases can be caused by Ustilago maydis infection ${ }^{39}$. Moreover, root-endophytic microbial communities have been shown to be linked to apple replant disease ${ }^{40}$. In this study, the occurrence of Verticillium, which was classified as an endophyte, also significantly increased with increased duration of continuous cropping of Tibet barley (Figure 6B). Verticillium dahliae not only delayed, but also reduced wilt symptoms in cotton ${ }^{41}$. Thus, Verticillium should function as beneficial fungi for the roots of Tibet barley. However, the effects of rhizosphere pathogens and endophytes on plants have not yet been examined during continuous cropping of Tibet barley; accordingly, future studies should be conducted to elucidate the mechanisms through which their effects occur.

\section{Conclusion}

In summary, the dynamics of fungal community structure and function during 6 years of continuous monoculture of Tibet barley, an important highland crop, were examined. 18S rRNA gene high-throughput sequencing was applied to obtain the fungal sequences and provide detailed insight into the distribution patterns of fungal communities in Tibet barley rhizosphere soil during continuous cropping. We found that continuous cropping of Tibet barley markedly reduced the fungal richness and phylogenetic diversity. Moreover, the plant-pathogen fungal genera Parastagonospora and Ustilago were enriched during continuous cropping. However, Verticillium, which should benefit plant roots and reduce wilt symptoms, increased. In summary, our research helps fill gaps in knowledge regarding the response of fungal communities to continuous cropping of plants cultivated in areas with high altitude, but further research is needed to elucidate the mechanisms of changes in abundance of fungal pathogens and endophytes in rhizosphere soil of Tibet barley.

\section{Methods}

\section{Study site description and soil sampling}

The Tibet-barley field was located at the Quankou Experimental Station, Menyuan Hui Autonomous County, Qinghai province, China $\left(37^{\circ} 21^{\prime} \mathrm{N}, 101^{\circ} 44^{\prime} \mathrm{E}\right)$. The experimental field has a mean annual precipitation of $530-560 \mathrm{~mm}$, an average annual sunshine duration of $2,575 \mathrm{~h}$, an average annual temperature of $1.3^{\circ} \mathrm{C}$, and an annual evaporation of $1,128-1,343 \mathrm{~mm}$. The experimental fields contained four plots, and each plot was $10.0 \times 10.0 \mathrm{~m}$ in size. Tibetan barley (Hordeum vulgare L., qingke) was cultivated each year in the field and from June 2011 to June 2016. Blended fertilizer with $200 \mathrm{mg} \mathrm{m}^{-2}$ $\left(\mathrm{NH}_{4}\right)_{2} \mathrm{SO}_{4}$ and $83 \mathrm{mg} \mathrm{m}^{-2}$ urea was applied annually before the seeding stage. Other field management activities were performed according to the practices of local farmers. Rhizosphere soil samples were collected annually during the flowering period. Approximately 15 plant samples were collected from five 
different sites using a " $Z$ " pattern in each experimental plot and then made into composite samples. Rhizosphere soils were collected for analysis as previously described ${ }^{42}$. Briefly, plants were carefully pulled from the ground, after which the conglutinating soil from the roots was mildly crushed and shaken to collect the soil located within the plant root surfaces. Quadruple soil and plant samples were collected from the four experimental fields. The rhizosphere soil was immediately transported on ice to the laboratory, where it was stored at $-20^{\circ} \mathrm{C}$ until DNA extraction and chemical analysis.

\section{Soil DNA extraction, PCR amplification, and Illumina MiSeq sequencing}

Community DNA was extracted using a MoBio Soil DNA isolation kit (MO BIO Laboratories Inc., USA) according to the manufacturer's protocols. The 18S rRNA gene was amplified using the fungal-specific primer pair NS1 (5'-GTA GTC ATA TGC TTG TCT C-3') and Fung (5'-ATT CCC CGT TAC CCG TTG-3') ${ }^{43}$ with a 12-base unique barcode attached to the forward primer. PCR reactions and amplicon purification were performed as described by Cheung et al. ${ }^{44}$. Finally, the purified products were quantified using a Qubit 3.0 (Thermo Fisher Scientific, USA) and then sequenced on an Illumina MiSeq system at Sangon Biotech Co., Ltd. (Shanghai, China).

\section{Processing of sequencing data}

Quantitative Insights into Microbial Ecology (QIIME) ${ }^{45}$ was applied to process the $18 \mathrm{~S}$ rRNA gene sequences after paired-end fragments were merged by $\mathrm{FLASH}^{46}$. Briefly, the demux plugin (https://github.com/qiime2/q2-demux) was used to demultiplex and classify sequences into the different samples by unique barcode. The q2-feature-classifier plugin (https://github.com/qiime2/q2-dada2) was then used for quality control and chimeric sequences removal with the default settings. Next, UCLUST ${ }^{47}$ was applied to cluster sequences into operational taxonomic units (OTUs) at $97 \%$ similarity. The classifier-sklearn plugins (https://github.com/qiime2/q2-feature-classifier) were used for taxonomic assignment of each sequence with the UNITE database ${ }^{48}$. The OTU table was rarefied to the minimum sample count (38,031 sequences for each sample) to reduce the effects of sequence numbers on diversity calculation.

The Shannon and Simpson's indices were calculated to represent alpha species diversity in the fungal community. Chao1 was used to describe the fungal species richness, and the PD index was used to determine the phylogenetic diversity ${ }^{49,50}$. To compare the fungal community beta diversity, Principal Coordinates Analysis ( $\mathrm{PCOA}$ ) was performed using the phyloseq package ${ }^{51}$ and plotted with the ggplot2 package $^{52}$.

\section{Identification of fungal taxa shared in rhizosphere soil during continuous cropping}

Taxa shared across samples were obtained from the taxa-abundance matrix (OTU table) generated by the QIIME software at the genus level. Dominant and rare OTUs in sequence libraries and shared taxa were defined at a threshold of $1 \%$ relative abundance ${ }^{53}$. Shared taxa in samples were visualized using the 
Pheatmap package in $\mathrm{R}^{54}$. For phylogenetic analysis of shared taxa at the genus level, representative sequences belonging to the OTUs of each shared taxon were extracted using QIIME and taxonomically classified after re-alignment with the UNITE database.

\section{Predicting function of fungal community in rhizosphere soil}

FUNGuild (https://omictools.com/fungi-functional-guild-tool) was applied to taxonomically parse fungal OTUs based on their ecological functional characteristics ${ }^{55}$, and only guild confidence rankings assigned to"highly probable" and "probable" were kept. We classified three fungal functional groups according to trophic mode: symbiotrophy, saprotrophy, and pathotrophy. The OTUs that could not be identified into functional groups were categorized as unclassified and are not present in our results description. The relative abundance was estimated with the data of reads for each functional group by dividing the total reads assigned by FUNGuild. Fungal OTUs belonging to multiple functional groups were also treated as members of other groups. The taxonomic information describing OTUs assigned as functional guilds was classified after re-alignment with the UNITE database using the QIIME software.

\section{Statistical analysis}

Statistical analyses were conducted using SPSS 18.0 software (SPSS Inc., Chicago, IL, USA). Statistical significance was estimated by two-way analysis of variance followed by the least significant difference test to determine the significance of differences between groups. Differences were considered to be significant when the $P$-value was less than 0.05 .

\section{Nucleic acid sequences}

The 18S rRNA gene sequences were deposited into the NCBI Sequence Read Archive with the accession number PRJNA669607.

\section{Declarations}

\section{AUTHOR CONTRIBUTIONS}

Yao-YH and Zhao-Y carried out the molecular experiments, analyzed data, and wrote the manuscript. Yao$\mathrm{YH}$ and Yao-XH carried out data analyses. Bai-YX, An-LK and Li-Xin contributed to the field experiments and collected samples. Wu-KL conceived the study, contributed to the design, and interpretation of the research. All authors read and approved the final manuscript.

\section{CONFLICTS OF INTERESTS}

The authors declare that the research was conducted in the absence of any commercial or financial relationships that could be construed as a potential conflict of interest.

\section{ACKNOWLEDGMENTS}


This work was supported by the Natural Science Foundation of China (32060447,31660388, and 31960427) and China Agriculture Research System (CARS-05). The authors are grateful to ECOGENE BIOTECH CO., LTD. (Shenzhen, China) for the support on bioinformatics of 18S rRNA amplicon data. We also thank LetPub (www.letpub.com) for its linguistic assistance during the preparation of this manuscript.

\section{References}

1 Dick, R. P. A review: Long-term effects of agricultural systems on soil biochemical and microbial parameters. Agric. Ecosyst. Environ. 40, 25-36 (1992).

2 Mohanram, S. \& Kumar, P. Rhizosphere microbiome: Revisiting the synergy of plant-microbe interactions. Ann. Microbiol. 69, 307-320 (2019).

3 Alami, M. M. et al. Structure, function, diversity, and composition of fungal communities in rhizospheric soil of Coptis chinensis Franch under a successive cropping system. Plants 9, 244 (2020).

4 Bai, L., Cui, J. Q., Jie, W. G. \& Cai, B. Y. Analysis of the community compositions of rhizosphere fungi in soybeans continuous cropping fields. Microbiol. Res. 180, 49-56 (2015).

5 Gao, Z. Y. et al. Effects of continuous cropping of sweet potato on the fungal community structure in rhizospheric soil. Front. Microbiol. 10 (2019).

6 Liu, J. J. et al. Continuous cropping of soybean alters the bulk and rhizospheric soil fungal communities in a Mollisol of Northeast PR China. Land Degrad. Dev. 30, 1725-1738 (2019).

7 Song, X. H., Pan, Y., Li, L. Y., Wu, X. L. \& Wang, Y. Composition and diversity of rhizosphere fungal community in Coptis chinensis Franch. continuous cropping fields. PLoS One 13, e0193811 (2018).

8 Qin, S. H., Yeboah, S., Xu, X. X., Liu, Y. H. \& Yu, B. Analysis on fungal diversity in rhizosphere soil of continuous cropping potato subjected to different furrow-ridge mulching managements. Front. Microbiol. 8, 845 (2017).

9 Xiong, W. et al. Different Continuous cropping spans significantly affect microbial community membership and structure in a Vanilla-grown soil as revealed by deep pyrosequencing. Microb. Ecol. 70, 209-218 (2015).

10 Haddaway, N. R., Styles, D. \& Pullin, A. S. Environmental impacts of farm land abandonment in high altitude/mountain regions: A systematic map of the evidence. Environ. Evid. 2, 18 (2013).

11 Kumar, S., Suyal, D. C., Yadav, A., Shouche, Y. \& Goel, R. Microbial diversity and soil physiochemical characteristic of higher altitude. PLoS ONE 14, e0213844 (2019). 
12 Rawat, J., Yadav, N. \& Pande, V. in Recent Advancements in Microbial Diversity (eds Surajit De Mandal \& Pankaj Bhatt) 147-196 (Academic Press, 2020).

13 Bryant, J. A. et al. Microbes on mountainsides: Contrasting elevational patterns of bacterial and plant diversity. Proc. Natl. Acad. Sci. U S A 105, 11505-11511 (2008).

14 Wang, J. T. et al. Altitudinal distribution patterns of soil bacterial and archaeal communities along mt. Shegyla on the Tibetan Plateau. Microb. Ecol. 69, 135-145 (2015).

15 Liu, H. et al. Response of soil fungal community structure to long-term continuous soybean cropping. Front. Microbiol. 9, 3316 (2019).

16 Li, W. h. \& Liu, Q. z. Changes in fungal community and diversity in strawberry rhizosphere soil after 12 years in the greenhouse. J. Integr. Agric. 18, 677-687 (2019).

17 Li, X. G., Ding, C. F., Zhang, T. L. \& Wang, X. X. Fungal pathogen accumulation at the expense of plant-beneficial fungi as a consequence of consecutive peanut monoculturing. Soil Biol. Biochem. 72, 1118 (2014).

18 Li, Y. C., Li, Z. W., Arafat, Y. \& Lin, W. X. Studies on fungal communities and functional guilds shift in tea continuous cropping soils by high-throughput sequencing. Ann. Microbiol. 70, 7 (2020).

19 Zhou, X. G. \& Wu, F. Z. Dynamics of the diversity of fungal and Fusarium communities during continuous cropping of cucumber in the greenhouse. FEMS Microbiol. Ecol. 80, 469-478 (2012).

20 Guedes, J. D., Lu, H. J., Hein, A. \& Schmidt, A. H. Early evidence for the use of wheat and barley as staple crops on the margins of the Tibetan plateau. Proc. Natl. Acad. Sci. U S A 112, 5625-5630 (2015).

21 Zeng, X. Q. et al. Origin and evolution of qingke barley in Tibet. Nat. Commun. 9, 5433-5433 (2018).

22 Zhao, X. Y., Wang, W. J., Wan, W. Y. \& Li, H. Influence of climate change on potential productivity of naked barley in the Tibetan Plateau in the past 50 years. Zhongguo Sheng Tai Nong Ye Xue Bao 23, 13291338 (2015).

23 Nguyen, N. H. et al. FUNGuild: An open annotation tool for parsing fungal community datasets by ecological guild. Fungal Ecol. 20, 241-248 (2016).

24 Arafat, Y. et al. Long-term monoculture negatively regulates fungal community composition and abundance of tea orchards. Agronomy 9, 466 (2019).

25 Dong, L. et al. High-throughput sequencing technology reveals that continuous cropping of American ginseng results in changes in the microbial community in arable soil. Chinese Medicine 12, 18 (2017). 
26 Singh, B. K. et al. Loss of microbial diversity in soils is coincident with reductions in some specialized functions. Environ. Microbiol. 16, 2408-2420 (2014).

27 Maron, P.-A. et al. High microbial diversity promotes soil ecosystem functioning. Appl. Environ. Microbiol. 84, e02738-02717 (2018).

28 Stursova, M., Žifcakova, L., Leigh, M. B., Burgess, R. \& Baldrian, P. Cellulose utilization in forest litter and soil: Identification of bacterial and fungal decomposers. FEMS Microbiol. Ecol. 80, 735-746 (2012).

29 Ludley, K. E. \& Robinson, C. H. 'Decomposer' Basidiomycota in Arctic and Antarctic ecosystems. Soil Biol. Biochem. 40, 11-29 (2008).

30 Wakelin, S. A., Warren, R. A., Kong, L. \& Harvey, P. R. Management factors affecting size and structure of soil Fusarium communities under irrigated maize in Australia. Appl. Soil Ecol. 39, 201-209 (2008).

31 Blagodatskaya, E. \& Kuzyakov, Y. Active microorganisms in soil: Critical review of estimation criteria and approaches. Soil Biol. Biochem. 67, 192-211 (2013).

32 Rouphael, Y. et al. Arbuscular mycorrhizal fungi act as biostimulants in horticultural crops. Sci. Hortic. 196, 91-108 (2015).

33 Igiehon, N. O. \& Babalola, O. O. Biofertilizers and sustainable agriculture: Exploring arbuscular mycorrhizal fungi. Appl. Microbiol. Biotechnol. 101, 4871-4881 (2017).

34 Anthony, M. A., Frey, S. D. \& Stinson, K. A. Fungal community homogenization, shift in dominant trophic guild, and appearance of novel taxa with biotic invasion. Ecosphere 8, e01951 (2017).

35 Feng, T. et al. Effects of wheat and soybean stubbles on soil sickness in continuous cropping of cucumber. Allelopathy J. 39, 43-53 (2016).

$36 \mathrm{Fu}, \mathrm{H}$. D. et al. Effects of continuous tomato monoculture on soil microbial properties and enzyme activities in a solar greenhouse. Sustainability 9, 317 (2017).

37 Liu, X. et al. Microbial community diversities and taxa abundances in soils along a seven-year gradient of potato monoculture using high throughput pyrosequencing approach. PLoS One 9, e86610 (2014).

38 Solomon, P. S. et al. Mannitol is required for asexual sporulation in the wheat pathogen Stagonospora nodorum (glume blotch). Biochem. J. 399, 231-239 (2006).

39 Kahmann, R., Steinberg, G., Basse, C., Feldbrügge, M. \& Kämper, J. in Fungal Pathology 347-371 (Springer, 2000). 
40 Manici, L. M. et al. Relationship between root-endophytic microbial communities and replant disease in specialized apple growing areas in Europe. Appl. Soil Ecol. 72, 207-214 (2013).

41 Yuan, Y. et al. Potential of endophytic fungi isolated from cotton roots for biological control against Verticillium wilt disease. PLoS One 12, e0170557 (2017).

42 Li, W. H., Liu, Q. Z. \& Chen, P. Effect of long-term continuous cropping of strawberry on soil bacterial community structure and diversity. J. Integr. Agric. 17, 2570-2582 (2018).

43 Möhlenhoff, P., Müller, L., Gorbushina, A. A. \& Petersen, K. Molecular approach to the characterisation of fungal communities: Methods for DNA extraction, PCR amplification and DGGE analysis of painted art objects. FEMS Microbiol. Lett. 195, 169-173 (2001).

44 Cheung, M. K., Au, C. H., Chu, K. H., Kwan, H. S. \& Wong, C. K. Composition and genetic diversity of picoeukaryotes in subtropical coastal waters as revealed by 454 pyrosequencing. ISME J. 4, 1053-1059 (2010).

45 Caporaso, J. G. et al. QIIME allows analysis of high-throughput community sequencing data. Nat. Methods 7, 335-336 (2010).

46 Magoč, T. \& Salzberg, S. L. FLASH: Fast length adjustment of short reads to improve genome assemblies. Bioinformatics 27, 2957-2963 (2011).

47 Edgar, R. C. Search and clustering orders of magnitude faster than BLAST. Bioinformatics 26, 2460-2461 (2010).

48 Nilsson, R. H. et al. The UNITE database for molecular identification of fungi: Handling dark taxa and parallel taxonomic classifications. Nucleic Acids Res. 47, D259-D264 (2019).

49 Thukral, A. K. A review on measurement of alpha diversity in biology. Agric. Res. 54, 1-10 (2017).

50 Cadotte, M. W., Dinnage, R. \& Tilman, D. Phylogenetic diversity promotes ecosystem stability. Ecology 93, S223-S233 (2012).

$51 \quad$ Package 'phyloseq' (2013).

52 Wickham, H., Chang, W. \& Wickham, M. H. Package 'ggplot2'. Create Elegant Data Visualisations Using the Grammar of Graphics. Version 2, 1-189 (2016).

53 Gobet, A., Quince, C. \& Ramette, A. Multivariate cutoff level analysis (MultiCoLA) of large community data sets. Nucleic Acids Res. 38, e155-e155 (2010).

54 Package 'pheatmap' (2015). 
55 Sun, J. Y. et al. Fungal community dynamics during a marine dinoflagellate (Noctiluca scintillans) bloom. Mar. Environ. Res. 131, 183-194 (2017).

Figures

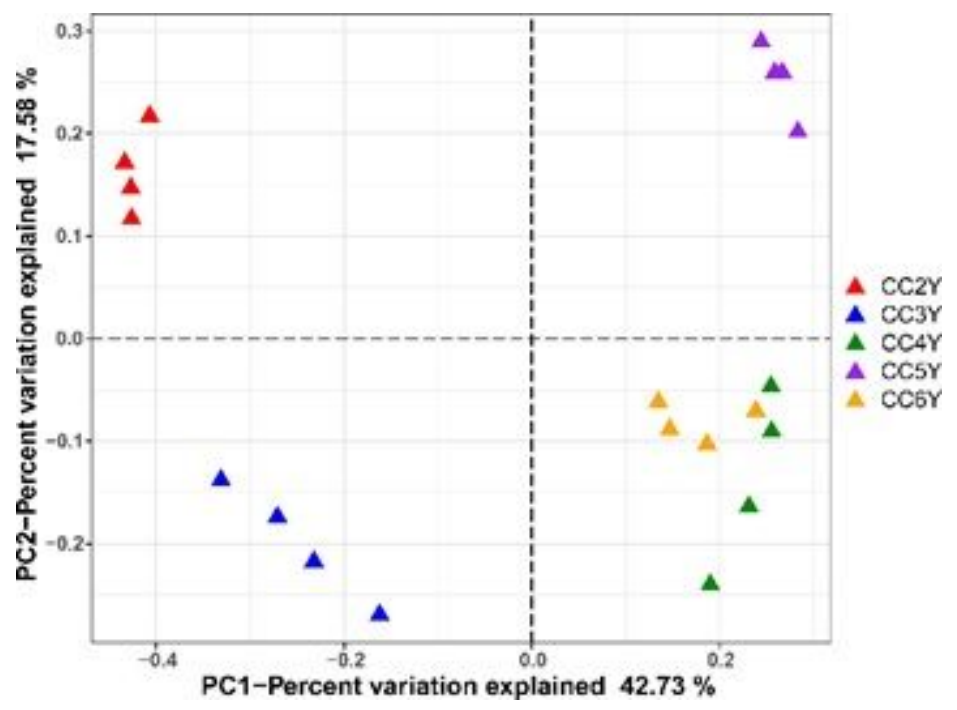

Figure 1

PCoA of fungal communities in rhizosphere soil samples based on Bray-Curtis distances. CC2Y, CC3Y, CC4Y, CC5Y, and CC6Y represent continuous cropping for 2, 3, 4, 5, and 6 years, respectively. The percent variation of the plotted principal component is indicated on the axes.
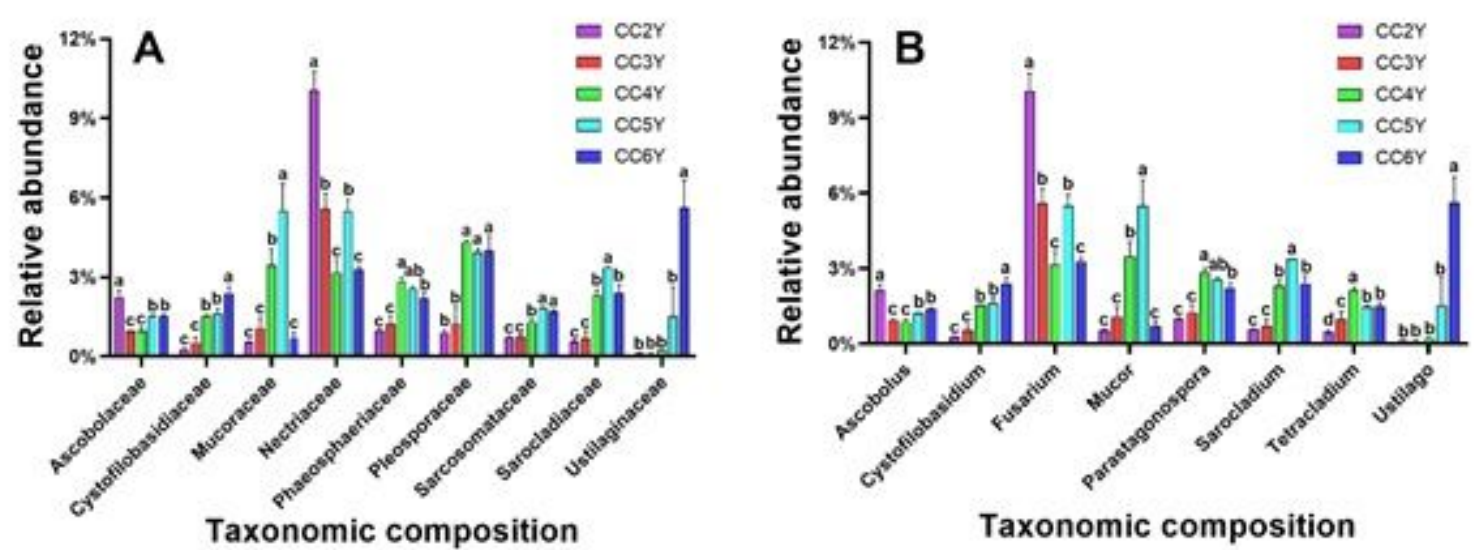

Figure 2

Relative abundance of fungal taxa in rhizosphere soil of Tibetan barley with different continuous cropping durations. (A) Relative abundances of the ten most abundant fungal families. (B) Relative abundances of the ten most abundant fungal genera. Symbols represent means $(n=4)$. 


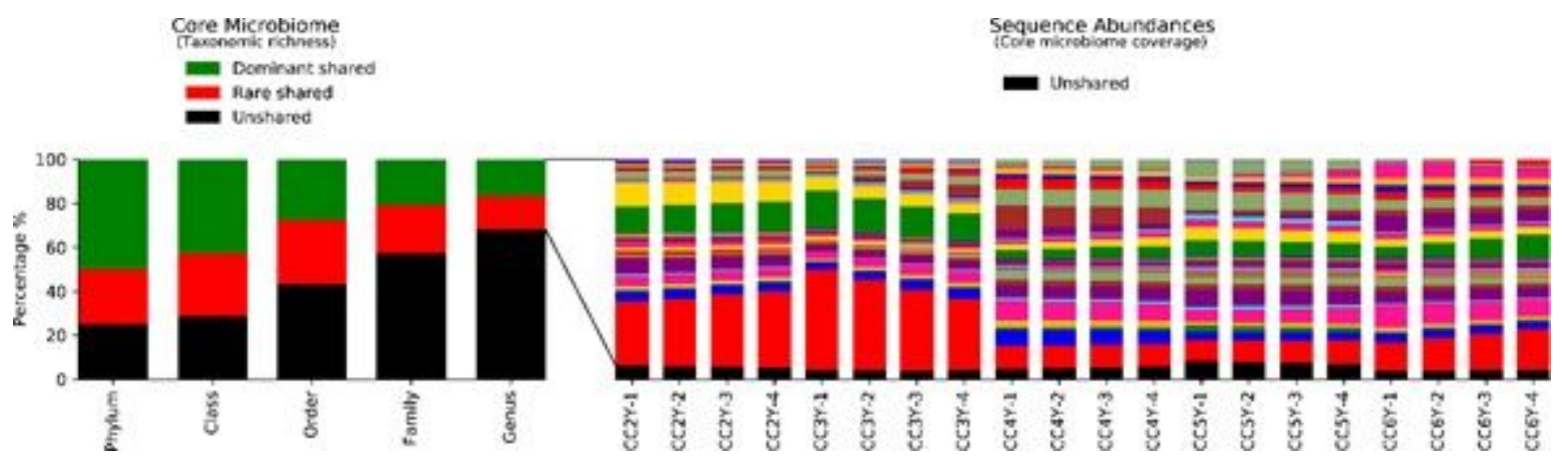

Figure 3

Richness and sequence coverage of shared taxa in rhizosphere fungal communities with different years of continuous cropping.

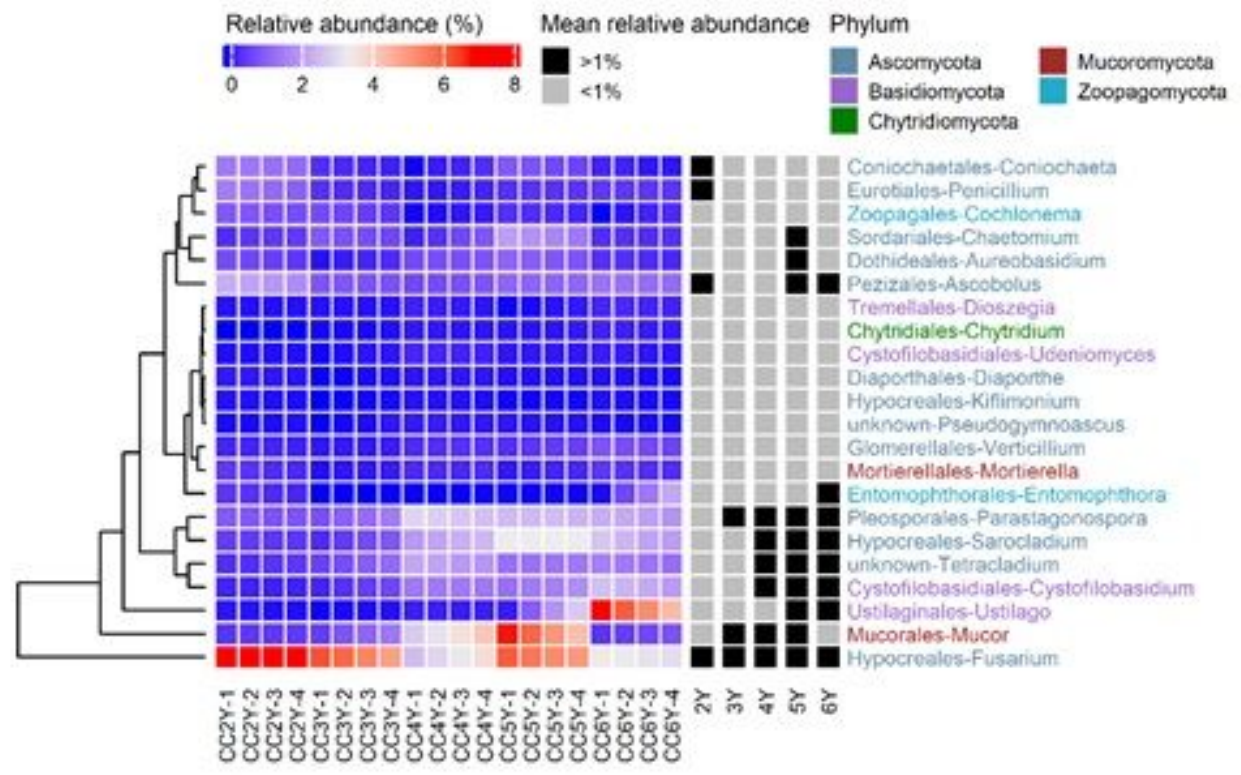

Figure 4

Shared genera and their absolute abundance. Sequence abundances were log-transformed and colored from red-to-blue to indicate higher-to-lower abundance, respectively.
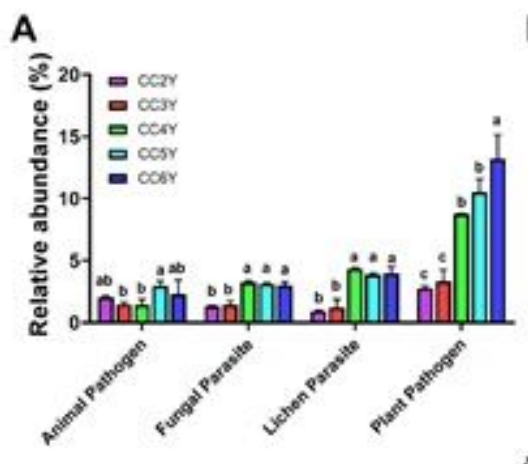
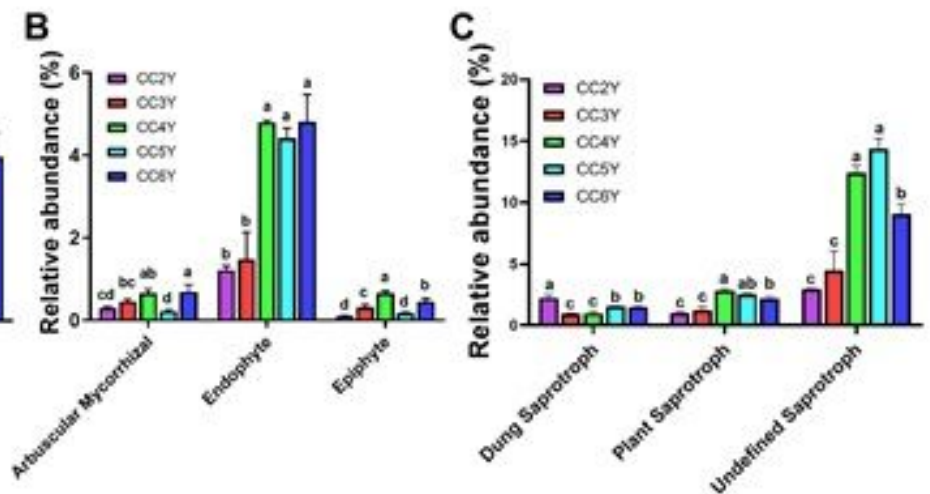

Figure 5 
Dynamics of trophic models in pathotrophs (A), symbiotrophs (B), and saprotrophs (C) during continuous cropping of Tibetan barley. Values represent means \pm standard deviation $(n=4)$. Different lowercase letters within the same column indicate significant differences among different continuous cropping durations at $\mathrm{P}<0.05$ according to two-way analysis of variance.
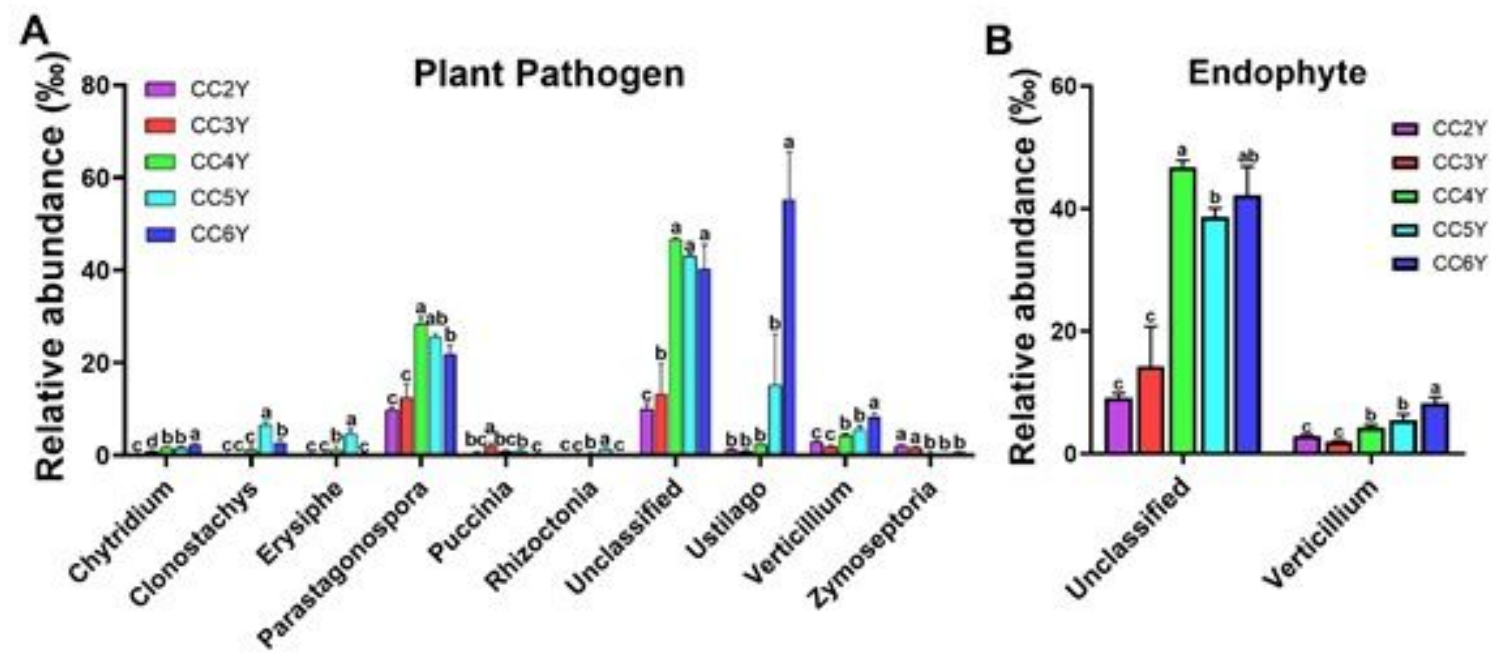

\section{Figure 6}

Taxonomic composition of fungi classified as plant pathogens (A) and endophytes (B) during continuous cropping. Values represent means \pm standard deviation $(n=4)$. Different lowercase letters within the same column indicate significant differences among different continuous cropping durations at $P<0.05$ according to two-way analysis of variance.

\section{Supplementary Files}

This is a list of supplementary files associated with this preprint. Click to download.

- FigureS1.Phylumcomposition.tif

- FigureS2.ThreeTrophicmodes.tif 